\title{
An Observational Exploration of Factors Affecting Perceived Social Isolation Among Social Media Using Medical Professional Course Students In South Indian State of India.
} Sree Sucharitha Tirukkovalluri ${ }^{1}$, Karthick Malarvannan ${ }^{2}$, Recharla Chenchu Karthik ${ }^{3}$, Balaji Sivaram Mahendiran ${ }^{4}$, Balaji Arumugam ${ }^{5}$

${ }^{1}$ Professor, Department of Community Medicine, Tagore Medical College and Hospital, Chennai -600127 ; ${ }^{2} 2$ nd Year MBBS Student, Tagore Medical College And Hospital, Chennai - 600127; ${ }^{3}$ Assistant Professor, Department of Community Medicine, Tagore Medical College and Hospital, Chennai - 600127; ${ }^{4}$ Assistant Professor, Department of Community Medicine, Madras Medical College and Hospital, Chennai -600003 ; ${ }^{5}$ Professor and Head, Department of Community Medicine, Tagore Medical College and Hospital, Chennai -600127

\begin{tabular}{|c|c|c|c|c|c|c|c|c|}
\hline Abstract & Introduction & Methodology & Results & Conclusion & References & Citation & \multicolumn{2}{|c|}{ Tables / Figures } \\
\hline \multicolumn{9}{|c|}{ Corresponding Author } \\
\hline \multicolumn{8}{|c|}{$\begin{array}{l}\text { Dr. S.M. Balaji, Department of Community Medicine, Madras Medical College and Hospital, Chennai - } \\
600003 \\
\text { E mail ID: drbalajism@gmail.com }\end{array}$} & 回部回 \\
\hline
\end{tabular}

\section{Citation}

Tirukkovalluri SS, Malarvannan K, Karthik RC, Mahendiran BS, Arumugam B. An Observational Exploration of Factors For Perceived Social Isolation Among Social Media Using Medical Professional Course Students In Chennai, India. Indian J Comm Health. 2020;32(1):76-81.

Source of Funding: Nil Conflict of Interest: None declared

Article Cycle

Received: 27/11/2019; Revision: 15/02/2020; Accepted: 05/03/2020; Published: 31/03/2020

This work is licensed under a Creative Commons Attribution 4.0 International License.

\section{Abstract}

Background: Social isolation is identified as a state in which individuals lack a sense of belonging, true engagement with immediate family, friends, peers in the form of fulfilling relationships. The subjective form 'perceived social isolation'(PSI) arises from a feeling of lack of engagement with above is linked to adverse physical and mental conditions. Aim \& Objectives: To assess the factors associated with social medial use and levels of perceived social isolation among study subjects. Materials \& Methods: PSI was assessed by using Patient-Reported Outcomes Measurement Information System (PROMIS) which was adapted in this study and the scores and grades from PROMIS-4 were classified as: low PSI: 4-6, medium PSI: 7-10, high PSI: 11 and above. Results: PSI was significantly higher among the subjects using social media for than two hours per day. In our study the association between gender, time spent on social media on daily basis, year of studying and PSI scores was found to be statistically significant. (P-value $>0.05$ ). Conclusion: People with high social media usage were found to perceive being socially isolated than their counterparts with lower use.

\section{Keywords}

Social Isolation, PROMIS tool, Social Media, Health Care Professional, Mental Health.

\section{Introduction}

Perceived social isolation (PSI) is a state in which individuals lack a sense of belonging, true engagement with immediate family, friends, peers in the form of fulfilling relationships. $(1,2)$ The 'objective' forms is actual lack of social ties with social systems such as family, community belonging etc.(3). The subjective form i.e. 'perceived social isolation' arises from a feeling of lack of engagement with systems available and linked to adverse physical and mental conditions in individuals experiencing it. $(2,4,5,6)$ The young adults aged 18-24 year's undergo transitional phase into adulthood and physical support systems and social networks provide immense strength to successful transition. The perceived social isolation among young adults finds its roots established when they move away from these protective systems. Global studies identified increased social support $(7,8)$ as well as depression and other mental health disorders $(9,10,11,12)$ among young adults who are regular social media users. Medical or health care professional (HCP) course students are at risk of intense stress owing to curriculum demands and are highest users of social media. Need arises to study their perceived state of social isolation to identify solutions and support systems before the impact of perceived social isolation leaks into adulthood. 


\section{Aims \& Objectives}

To assess the factors associated with social medial use and levels of perceived social isolation among health care professional course students with social media use.

\section{Material \& Methods}

Study Type: A cross- sectional, descriptive study was conducted in South Indian metro city, Chennai

Study Population: HCP course students who were habitual social media users, enrolled in a tertiary medical teaching hospital for academic courses including medicine, dental, nursing, allied health sciences

Study Area: Tertiary care teaching hospital, in a South Indian metro city, Chennai during October-December 2019.

Study Duration: October-December, 2019.

Sample Size Calculation: Based on Primack et al study the prevalence $-27 \%$ (p), 3.5\%(d) allowable error, and $10 \%$ non-response rate, and considering the confidence interval of 95\%, sample size was calculated to be 708 .

Inclusion Criteria: HCP course students, aged 17-24 years from both genders, consenting and willing to complete the questionnaire.

Exclusion Criteria: Students who are not habitual social media users were not included in the study.

Strategy for collection: A pre-tested, structured questionnaire was designed to collect data on sociodemographic characteristics, and survey items to assess social media usage such as duration of time spent on social media and frequency of usage in a day. Perceived social isolation is assessed by items adapted from global validated tool-Patient-Reported Outcomes Measurement Information System [PROMIS] (13) This tool development was an NIH Roadmap initiative which aims to provide precise, valid, reliable, and standardized questionnaires across the domains of physical, mental, and social health and measures patient reported outcomes. The PROMIS social isolation scale has been correlated with and validated against other commonly used social isolation measures. $(14,15)$ In a 2018 published study PROMIS social isolation scale has been successfully adopted to measure perceived social isolation in a nationally representative sample of university going adults in United States by Brian A. Primack et al. (16) The items in the scale, specifically ask of the participants, how frequently during the past seven days: i. they had felt left out, ii. that people barely knew them, iii. felt isolated from others, iv. felt people are around but not with them. These items were scored on a 5-point Likert Scale ranging from 1 to 5, and correspond to never (score=1), rarely (score=2), sometimes (score=3), often and always (score=4). The cumulative scores range from 4 to 20 and are classified as: i. low PSI= total core between 4-6, medium PSI= total score between 7-10, high PSI as 11 and above. The students were approached at hostels, canteen and also at common rooms during lunch breaks, evenings and willing students were included in the study.

Working Definition: HCP course students of Tagore Medical college Hospital, Chennai currently admitted in professional courses, and are habitual i.e., at least once in a day for a duration of 15-30 minutes) were eligible to participate in the study with consent.

Ethical Approval: Institutional Ethics Committee (IEC) of Tagore Medical College and Hospital, Chennai approved the study protocol.

Consent: Written informed consent was obtained and the questionnaire was self-administered after the study participants fulfill the inclusion criteria and verification of identity card as proof of their HCP course status.

\section{Data Analysis}

Data was entered in Standard Microsoft Word Excel (2007 Version) and statistical analysis was performed on SPSS computer package version 21.0 (SPSS Inc.II, USA). The descriptive statistics were measured and differences between social media use and levels of perceived social isolation were assessed by Chi-Square test and a significance level of $p<0.05$ was set.

\section{Results}

Characteristics of study participants: A total of $911 \mathrm{HCP}$ course students in a tertiary medical teaching hospital, Chennai completed the survey questionnaires and data was analyzed. Majority (60.9\%) were females and $69.8 \%$ were aged 17-21 years.

As noted in (Table 1), the descriptive features of professional course students was depicted.

(Table 2) shows the grades of PSI distributed among participants and Low PSI (4-6) was found in 495 (54.3\%), Medium PSI (7-10) in 148(16.2\%), High PSI (11and above) in $286(29.4 \%)$.

In (Table-3), participants aged 17-21 years had high perceived isolation when compared to other higher age groups. The association between age and perceived social isolation was found to be statistically significant. ( $P$ value $=0.0001$ ). Also statistically significant association was found between gender and PSI scores. (Pvalue $=0.0001$ ). Medium and high levels of perceived social isolation were high among female students across HCP courses when compared to males. The association between education, year of studying with perceived social isolation was found to be statistically significant. ( $P$ value=less than 0.05).

(Table-4) A statistically significant association was found between use of social media to make friends and PSI (Pvalue=0.002).

(Table 5) shows the association between time spent on social media for more than two hours on daily basis and high levels of perceived social isolation to be statistically significant. (P-value=0.029). In our study social media use across health care professional course students was found 
to be approximately $98.2 \%$. Only 16 individuals (1.8\%)

reported zero site visits per week.

In (Table-6), study participants with high Whatsapp usage

had 1.72 times higher risk of experiencing high PSI compared to others with lower usage of Whatsapp $(p<$ 0.05).

\section{Discussion}

Among health care professional course students of medical, dental, nursing and allied health sciences, we attempted to understand perceived social isolation among habitual social media users in Chennai.

\section{Use of social media among HCP course students}

This being a descriptive, cross-sectional study we narrated the social media use of various popular social media platforms by the study participants, as well as the duration of use. In our study, social media use across health care professional course students was found to be approximately $98.2 \%$. (Table 5) Similar studies reported that Social media use among Indian medical students was estimated to be $90 \%(17)$ and $88.58 \%(18)$ ). It was also found that medical students have higher prevalence of social media use compared to paramedical course students (18) which is similar to our finding in this study. In Kolkata study among medical undergraduates, it was reported that more than $90 \%$ use more than one social networking medium and one-third remain active all through the day and $80 \%$ for $\geq 4$ hours (19). In the same study, $24 \%$ reported depression and $68.5 \%$ had anxiety.

\section{Perceived Social Isolation among social media users}

Globally mixed findings of both positive and negative influences of social media usage on the mental health of the users have been widely debated. This is first study to our knowledge assessing social media use and perceived social isolation in India. Perceived social isolation variedly referred in many studies as 'loneliness' was first described by Hawkley LC et al in 2008 as a mismatch between an individual's social needs and the provisions offered in the social environment or is perceived to offer. The mismatch can be quantitative (i.e. too few relationships, potential or actual), but is described as usually more qualitative and subjective in interpretation leading to failure to arrive at a common definition (20). Isolation impacts social relationships resulting in failure to establish meaningful connections and bonding essential for survival of human beings. The various forms of isolation perceived by subjects have transient to long lasting mental health effects manifesting as depression, anxiety, withdrawal from active social life and could be fatal leading to suicides. Ecological systems theory as described Bronfenbrenner helps us to understand the fluid nature of forming relationships based on existing, current environmental constraints.(21) Wider interest in understanding the impact of social media use on spectrum of health ranging from physical, social, emotional and cognitive dimensions were attempted among adolescents and young college going adults (22-25). Our study contributed to expand the literature by understanding the influence of social media on the psychosocial wellbeing and it was found that PSI was significantly higher among those subjects who were using social media for than two hours in a day. They had nearly double the odds for experiencing PSI when compared to those using less than half hour in a day. Similar findings were reported in a study done in US based young adult population by Primack et al.(16) It also highlighted that the higher frequency of visiting the sites(58 times in a week) tripled the odds for PSI than fewer site visits (9 times a week).The researchers offered multiple theories aiding for PSI including social media use eating into real life relationship building, experiences of exclusion after coming across pictures shared from highly glamourous events/places/people concluding that in their study use of social media is found to be associated with increased social isolation(16). Heavy users (vs. light) of digital media were $48 \%$ to $171 \%$ more likely to be unhappy, to be in low in well-being.(26,) Light users (rather than non- or moderate users) were highest in well-being, and for most digital media use the largest drop in well-being occurred between moderate use and heavy use. All professional course students including computer courses, have been subjects of interest for researchers and mimic similar findings even in culturally different contexts with digital literacy slowly expanding due to wider accessibility and affordable data packages. $(27,28,29,30)$.

Studies on PSI from India or south Asia are not found to our knowledge though in the study based in Kolkata, the authors concluded that impact of social media use on interpersonal relations was inconclusive (18). Interestingly they also reported that among participants who felt addicted to social media networking, $62.8 \%$ indicated that it has improved relations. It is our understanding that todays' youth are quick to adapt and build inherent resilience systems of coping using social media platforms and this may have productive outcomes for this age group. In a correlational study among 467 young adults for their time spent using social media, results indicated that social media use was not predictive of impaired mental health functioning.(31) Also, authors concluded that after controlling for prior problems and loneliness, social networking sites (SNS) use does not or hardly predict mental health and sleep problems on the short or long term.(32)

\section{Conclusion}

The present study identified the levels of perceived social isolation among health care professional student. Multiple social media use characteristics were found to be significantly associated with different levels of perceived social isolation. 


\section{Recommendation}

Authors believe the present extensive work done in understanding characteristics of social media use and impact on perceived social isolation, including more indepth study among young adults will provide evidence informed psychosocial support and cognitive behavior therapies aimed at prevention and effective management of it.

\section{Limitation of the study}

The self-reported data on social media use and perceived social isolation from the study participants might not be amenable for generalization of data across different settings. But we believe it still aids to document the trends of the same. This study focused only on health care professional course students and further studies involving other academic course students will help reveal comprehensive understanding of social media use and perceived social isolation among college youth. Further studies should explore in detail about perceived social isolation.

\section{Relevance of the study}

Studies exploring mental and emotional well-being of health care professional students is extremely important to design promotive, preventive, support and rehabilitative systems in the campus and we see increasing trends in suicides in recent times across medical schools.

\section{Authors Contribution}

STS conceived the idea of the manuscript, study tools, organized the project, supervised the data collection and drafted the manuscript. KM co-ordinated data collection, data entry, data cleaning and data checks independently and under guidance of primary authors, KRC participated in finalizing data sheets for final analysis, BSM performed the data analysis and supported the manuscript assembling as per journal submission guidelines. All authors reviewed the final manuscript.

\section{Acknowledgement}

We acknowledge the support and participation in this survey of all the study participants from Tagore Medical College and Hospital, Chennai. We submit our sincere gratitude to Dr. I. Kannan, PhD, Co-ordinator, IRC, TMCH, Chennai.

\section{References}

1. Nicholson R. A review of social isolation: An important but underassessed condition in older adults. J Prim Prev 2012; 33(23):137-52.

2. Pantell M, Rehkopf D, Jutte D, Syme SL, Balmes J, Adler N. Social isolation: a predictor of mortality comparable to traditional clinical risk factors. Am J Public Health 2013; 103(11):2056-62.

3. Holt-Lunstad J, Smith TB, Baker M, Harris T, Stephenson D. Loneliness and social isolation as risk factors for mortality a metaanalytic review. PerspectPsychol Sci 2015; 10(2):227-37.
Cacioppo JT, Hawkley LC. Perceived social isolation and cognition. Trends Cogn Sci 2009; 13(10): 447-54. [Trends in Cognitive Sciences]

5. Holwerda TJ, Beekman AT, Deeg DJ, Stek ML, van Tilburg TG, Visser PJ. Increased risk of mortality associated with social isolation in older men: only when feeling lonely? Results from the Amsterdam Study of the Elderly (AMSTEL). Psychol Med 2012; 42(4):843-53. [Cambridge University Press].

6. Holwerda TJ, Deeg DJ, Beekman AT, van Tilburg TG, Stek ML, Jonker C. Feelings of loneliness, but not social isolation, predict dementia onset: results from the Amsterdam Study of the Elderly (AMSTEL). J Neurol Neurosurg Psychiatry 2014; 85(2):135-42

7. Steinfield C, Ellison NB, Lampe C. Social capital, self-esteem, and use of online social network sites: A longitudinal analysis. J Appl Dev Psychol 2008; 29:434-45.

8. Ellison NB, Steinfield C, Lampe C. The benefits of facebook "friends:" Social capital and college students' use of online social network sites. J Comput Commun 2007; 12(4):1143-68.

9. Kross E, Verduyn P, Demiralp E, Park J, Lee DS Facebook use predicts declines in subjective well-being in young adults. PLoS One 2013; 8(8): e69841.

10. Chou HTG, Edge N.They are happier and having better lives than am: The impact of using Facebook on perceptions of others' lives. Cyberpsychol Behav Soc Netw 2012; 15(2):117-21. https://doi.org/10.1089/cyber.2011.0324.

11. Sagioglou C, Greitemeyer T. Facebook's emotional consequences: Why Facebook causes a decrease in mood and why people still use it. Comput Human Behav 2014; 35:359-63.

12. Lin LY, Sidani JE, Shensa A, Radovic A, Miller E, Colditz JB Association between social media use and depression among U.S. young adults. Depress Anxiety 2016; 33(4):323-31.

13. Cella D, Riley W, Stone A, Rothrock N, Reeve B, Yount S The PatientReported Outcomes Measurement Information System (PROMIS) developed and tested its first wave of adult self-reported health outcome item banks: 2005-2008. J Clin Epidemiol 2010; 63(11):1179-94. [

14. Stacciarini JM, Smith R, Garvan CW, Wiens B, Cottler LB. Rural Latinos' mental wellbeing: A mixed-methods pilot study of family, environment and social isolation factors. Community Ment Health J 2015; 51(4):404-13.

15. Johnston KL, Lawrence SM, Dodds NE, Yu L, Daley DC, Pilkonis PA Evaluating $\mathrm{PROMIS}^{\circledR}$ instruments and methods for patientcentered outcomes research: Patient and provider voices in a substance use treatment setting. Qual Life Res 2016; 25(3):615-24.

16. Primack BA, Shensa A, Sidani JE, Whaite EO, Lin LY, Rosen D, et al. Social Media Use and Perceived Social Isolation Among Young Adults in the U.S Am J Prev Med 2017; 53(1): 1-8.

17. El Bialy $S$, Ayoub AR. The trends of use of social media by medical students. Education in medicine Journal. 2017; 9(1):59-68.

18. Lahiry S, Choudhury S, Chatterjee $S$, Hazra A. Impact of social media on academic performance and interpersonal relation: A crosssectional study among students at a tertiary medical center in East India. J Educ Health Promot 2019; 8: 73

19. Barman L, Mukhopadhyay DK, Bandyopadhyay GK. Use of Social Networking Site and Mental Disorders among Medical Students in Kolkata, West Bengal. Indian J Psychiatry 2018; 60(3): 340-5.

20. Hawkley LC, Hughes ME, Waite LJ, Masi CM, Thisted RA \& Cacioppo JT. From social structural factors to perceptions of relationship quality and loneliness: the Chicago Health, Aging, and Social Relations study. J Gerontol B Psychol Sci Soc Sci 2008; 63(6): S37584.

21. Bronfenbrenner, U. The Ecology of Human Development: Experiments by Nature and Design. Cambridge, MA: Harvard University Press

22. Azizi, S.M., Soroush, A. \& Khatony, A. The relationship between social networking addiction and academic performance in Iranian students of medical sciences: a cross-sectional study. BMC Psychol 7, 28 (2019). https://doi.org/10.1186/s40359-019-0305-0

23. Masthi NR, Pruthvi S, Phaneendra M. A comparative study on social media usage and health status among students studying in pre- 
INDIAN JOURNAL OF COMMUNITY HEALTH / VOL 32 / ISSUE NO 01 / JAN - MAR 2020 university colleges of urban Bengaluru. Indian journal of community medicine: official publication of Indian Association of Preventive \& Social Medicine. 2018;43(3):180.

24. Kumar S, Kumar A, Badiyani B, Singh SK, Gupta A, Ismail MB. Relationship of internet addiction with depression and academic performance in Indian dental students. Clujul Medical. 2018;91(3):300.

25. Madaiah M, Seshaiyengar CT, Suresh P, Munipapanna S, Sonnappa SD. Study to assess the effects of social networking sites on medical college students. International Journal of Community Medicine And Public Health. 2017;3(5):1204-8.

26. Bhatti A, Haq A. The Pathophysiology of Perceived Social Isolation: Effects on Health and Mortality. Cureus 2017; 9(1): e994.

27. Masters K. Social networking addiction among health sciences students in Oman. Sultan Qaboos Univ Med J. 2015;15(3): e357.

28. Ganapthi AVPRC. Internet addiction and associated factors: a study among college students in South India. Innovative Journal of Medical and Health Science. 2015;5(3):121-5.
[An Observational Exploration] | Tirukkovalluri SS et al

29. Ndasauka Y, Hou J, Wang Y, Yang L, Yang Z, Ye Z, et al. Excessive use of twitter among college students in the UK: validation of the microblog excessive use scale and relationship to social interaction and loneliness. Comput Hum Behav. 2016; 55: 963-71.

30. Twenge J \& Campbell WK. Media Use Is Linked to Lower Psychological Well-Being: Evidence from Three Datasets. Psychiatric Quarterly 2019; 90(2):311-31. doi: 10.1007/s11126019-09630-7.

31. Berryman C, Ferguson CJ, Negy C. Social Media Use and Mental Health among Young Adults. Psychiatr Q 2018; 89(2):307-14. doi: 10.1007/s11126-017-9535-6.

32. Van der Velden PG, Setti I, van der Meulen E \& Das M. Does social networking sites use predict mental health and sleep problems when prior problems and loneliness are taken into account? A population-based prospective study. Computers in Human Behavior 2019; 93: 200-9.

\section{Tables}

TABLE 1 DISTRIBUTION OF STUDY PARTICIPANTS ACCORDING TO HCP COURSES.

\begin{tabular}{|c|c|c|}
\hline Demographic factors & Frequency $(n=911)$ & Percentage \\
\hline \multicolumn{3}{|l|}{ Age } \\
\hline $17-21$ yrs & 636 & 69.8 \\
\hline 22 yrs and above & 275 & 30.2 \\
\hline \multicolumn{3}{|l|}{ Gender } \\
\hline Male & 356 & 39.1 \\
\hline Female & 555 & 60.9 \\
\hline \multicolumn{3}{|l|}{ Education } \\
\hline MBBS & 732 & 80.4 \\
\hline Nursing & 99 & 10.8 \\
\hline AHS* & 80 & 8.8 \\
\hline \multicolumn{3}{|l|}{ Year of Study } \\
\hline $1^{\text {st }}$ year & 433 & 47.5 \\
\hline $2^{\text {nd }}$ year & 186 & 20.4 \\
\hline $3^{\text {rd }}$ year & 140 & 15.4 \\
\hline $4^{\text {th }}$ year & 111 & 12.2 \\
\hline $5^{\text {th }}$ year & 41 & 4.5 \\
\hline
\end{tabular}

\section{TABLE 2 DISTRIBUTION OF PSI GRADING AMONG STUDY PARTICIPANTS}

\begin{tabular}{|l|c|c|c|}
\hline \multicolumn{1}{|c|}{ PSI GRADING } & SCORE & FREQUENCY & PERCENTAGE \\
\hline Low & $4-6$ & 495 & 54.3 \\
\hline Medium & $7-10$ & 268 & 29.4 \\
\hline High & 11 \& ABOVE & 148 & 16.2 \\
\hline *PSI: Perceived social isolation & & & \\
\hline
\end{tabular}

TABLE 3 ASSOCIATION BETWEEN DEMOGRAPHIC FACTORS AND PSI OF STUDY PARTICIPANTS

\begin{tabular}{|c|c|c|c|c|}
\hline \multirow[t]{2}{*}{ Variable } & \multicolumn{3}{|c|}{ PSI } & \multirow[t]{2}{*}{ Chi -square } \\
\hline & Low & Medium & High & \\
\hline \multicolumn{5}{|l|}{ 1.Age } \\
\hline $17-21$ years & 317 & 210 & 109 & \\
\hline 22 years and above & 178 & 58 & 39 & 0.0001 \\
\hline \multicolumn{5}{|l|}{ 2.Gender } \\
\hline Male & 281 & 48 & 27 & \\
\hline Female & 214 & 220 & 121 & 0.0001 \\
\hline \multicolumn{5}{|l|}{ 3.Education } \\
\hline MBBS & 389 & 220 & 123 & \\
\hline Nursing & 46 & 35 & 18 & 0.002 \\
\hline AHS & 60 & 13 & 7 & \\
\hline \multicolumn{5}{|l|}{ 4. Year of Study } \\
\hline 1 & 233 & 139 & 61 & \\
\hline
\end{tabular}




\begin{tabular}{|c|c|c|c|c|}
\hline 2 & 103 & 44 & 39 & 0.001 \\
\hline 3 & 79 & 39 & 22 & \\
\hline 5 & 47 & 39 & 25 & 1 \\
\hline
\end{tabular}

\section{TABLE 4 ASSOCIATION BETWEEN HCP COURSE AND SOCIAL MEDIA USE WITH PSI}

\begin{tabular}{|c|c|c|c|c|}
\hline \multirow[t]{2}{*}{ Variable } & \multicolumn{3}{|c|}{ PSI } & \multirow[t]{2}{*}{ Chi -square } \\
\hline & Low & Medium & High & \\
\hline \multicolumn{5}{|l|}{ 1. Social media usage frequency } \\
\hline Current user & 406 & 221 & 124 & \\
\hline Past & 10 & 9 & 8 & \\
\hline Rare & 61 & 31 & 11 & \\
\hline Never & 18 & 7 & 5 & 0.266 \\
\hline \multicolumn{5}{|c|}{ 2. Reasons for turning to social media $(n=880)$} \\
\hline To make more friends & 405 & 215 & 103 & \\
\hline Not to feel isolated/ lonely & 71 & 46 & 40 & 0.002 \\
\hline \multicolumn{5}{|l|}{ 3.I am a social media user $(n=881)$} \\
\hline Passive & 349 & 178 & 102 & \\
\hline Active & 128 & 83 & 41 & 0.361 \\
\hline \multicolumn{5}{|c|}{ 4. Social media usage contributes to my emotional well being } \\
\hline Unsatisfactory & 21 & 14 & 20 & \\
\hline Neutral & 283 & 146 & 69 & 0.001 \\
\hline Satisfactory & 173 & 101 & 54 & \\
\hline \multicolumn{5}{|c|}{ 5.Social media use makes me feel isolated/lonely } \\
\hline Often & 13 & 9 & 20 & \\
\hline Sometimes & 70 & 68 & 49 & 0.0001 \\
\hline Rarely or never & 394 & 184 & 74 & \\
\hline \multicolumn{5}{|c|}{ 6. Can cope up with social media use $\&$ its emotional side effects } \\
\hline Yes & 299 & 149 & 84 & \\
\hline No & 23 & 20 & 20 & 0.011 \\
\hline Don't know & 153 & 92 & 39 & \\
\hline
\end{tabular}

TABLE 5 ASSOCIATION BETWEEN SOCIAL MEDIA USAGE TIMEDAILY AND PERCEIVED SOCIAL ISOLATION (PSI)

\begin{tabular}{|c|c|c|c|c|c|}
\hline \multirow{2}{*}{$\begin{array}{c}\text { Time spent on social media on daily } \\
\text { basis }\end{array}$} & \multicolumn{3}{|c|}{ PSI score } & \multirow[t]{2}{*}{ Total } & \multirow[t]{2}{*}{ Chi -square } \\
\hline & Low & Medium & High & & \\
\hline $0-30 \mathrm{~min}$ & 43 & 24 & 12 & 79 & \multirow{5}{*}{$0.029 *$} \\
\hline $31-60 \mathrm{~min}$ & 54 & 22 & 10 & 86 & \\
\hline $61-120 \mathrm{~min}$ & 130 & 45 & 34 & 209 & \\
\hline $121 \mathrm{~min} \&$ above & 268 & 177 & 92 & 537 & \\
\hline Total & 495 & 268 & 148 & 911 & \\
\hline
\end{tabular}

TABLE 6 MULTIVARIATE ANALYSIS BETWEEN DIFFERENT SOCIAL MEDIA PLATFORMS AND PERCEIVED SOCIAL ISOLATION

\begin{tabular}{|c|c|c|c|}
\hline PSI SCORE & Variables & Adjusted Odds ratio & $P$ value \\
\hline \multirow[t]{7}{*}{ Medium } & Intercept & -- & $<0.001$ \\
\hline & Facebook & $1.26(0.75-2.11)$ & 0.384 \\
\hline & Whatsapp & $1.07(0.76-1.49)$ & 0.707 \\
\hline & Twitter & $0.79(0.32-1.95)$ & 0.610 \\
\hline & Instagram & $2.03(1.39-2.96)$ & $<0.001^{*}$ \\
\hline & YouTube & $0.9(0.59-1.35)$ & 0.599 \\
\hline & Others & $0.91(0.53-1.55)$ & 0.720 \\
\hline \multirow[t]{7}{*}{ High } & Intercept & -- & $<0.001$ \\
\hline & Facebook & $1.73(0.98-3.05)$ & $0.060 *$ \\
\hline & Whatsapp & $1.72(1.13-2.6)$ & $0.011^{*}$ \\
\hline & Twitter & $1(0.36-2.74)$ & 0.997 \\
\hline & Instagram & $0.91(0.56-1.46)$ & 0.682 \\
\hline & YouTube & $1.66(1.04-2.64)$ & $0.033^{*}$ \\
\hline & Others & $1.02(0.55-1.9)$ & 0.955 \\
\hline
\end{tabular}

